\title{
Semantic Relativism and Ways of Knowing
}

DOI:

10.1007/s11229-017-1572-z

\section{Document Version}

Accepted author manuscript

Link to publication record in Manchester Research Explorer

\section{Citation for published version (APA):}

Tarasov, L. (2017). Semantic Relativism and Ways of Knowing. Synthese. https://doi.org/10.1007/s11229-017$1572-z$

\section{Published in:}

Synthese

\section{Citing this paper}

Please note that where the full-text provided on Manchester Research Explorer is the Author Accepted Manuscript or Proof version this may differ from the final Published version. If citing, it is advised that you check and use the publisher's definitive version.

\section{General rights}

Copyright and moral rights for the publications made accessible in the Research Explorer are retained by the authors and/or other copyright owners and it is a condition of accessing publications that users recognise and abide by the legal requirements associated with these rights.

\section{Takedown policy}

If you believe that this document breaches copyright please refer to the University of Manchester's Takedown Procedures [http://man.ac.uk/04Y6Bo] or contact uml.scholarlycommunications@manchester.ac.uk providing relevant details, so we can investigate your claim.

\section{OPEN ACCESS}




\title{
Semantic Relativism and Ways of Knowing
}

By Leonid Tarasov

(forthcoming in Synthese)

\begin{abstract}
:
There is a long-standing view in epistemology that perception is a way of knowing (WOK). There is a less long-standing but increasingly popular view that knowledge attributions have a relativist semantics (RKA). I discuss three things here. First, I show that it is a consequence of the logic of RKA that WOK and RKA are incompatible. Second, I argue that, even if WOK is incompatible with the main rivals to RKA, this is not a consequence of the logics of these views. RKA comes with a hitherto unobserved philosophical consequence that its main rivals do not come with. Third, I consider some responses to the effect that it does not matter that RKA comes with this consequence, and argue that they are all unsatisfactory as they stand. I conclude that, at the very least, the onus is placed on relativists to engage with more epistemology than they currently do to show that we do not need to worry about the incompatibility of WOK and RKA. I conclude with some suggestions about the broader relevance and implications of this paper.
\end{abstract}

Key words: semantic relativism; ways of knowing; knowledge attributions; perception; assessment-sensitivity; logical implication.

\section{Introduction}

There is a long-standing view in epistemology that perception is a way of knowing. That is, for any subject $\mathrm{x}$ and any proposition $\mathrm{p}$, that $\mathrm{x}$ perceives that $\mathrm{p}$ logically implies that $\mathrm{x}$ knows that $\mathrm{p}$. For example, that $\mathrm{x}$ sees that $\mathrm{p}$ logically implies that $\mathrm{x}$ knows that p (see, e.g., Chisholm 1989: 41; Moore 1953: 77; Russell 1948: 422; Williamson 2000: 34). Call this the ways of knowing claim (WOK):

WOK: For any subject $\mathrm{x}$ and any proposition $\mathrm{p}$, that $\mathrm{x}$ perceives that $\mathrm{p}$ logically implies that $\mathrm{x}$ knows that $\mathrm{p}$. 
There is a less long-standing but increasingly popular view that knowledge attributions, i.e. sentences of the form $\ulcorner\mathrm{x}$ knows that $\Phi\urcorner$ and $\ulcorner\mathrm{x}$ does not know that $\Phi\urcorner,{ }^{1}$ are assessment-sensitive (see e.g. MacFarlane 2005; MacFarlane 2014: Ch. 8). Roughly, the truth-value of the proposition expressed by a knowledge attribution varies with respect to epistemic standards determined by contexts of assessment (this view will be explained in detail in §2). Call this semantic relativism about knowledge attributions (RKA):

$R K A$ : Knowledge attributions are assessment-sensitive.

I discuss three things here. First, I demonstrate that it is a consequence of the logic of RKA that WOK and RKA are incompatible. By the logic of a view I mean the set of formal principles that underlie the semantics given by that view. In particular, we will see that as long as we have the right number of parameters in so-called circumstances of evaluation and the values of these parameters vary in the way prescribed by relativist semantics, we get the result that RKA and WOK are incompatible. This variation in the values of and interaction between parameters of circumstances can be understood purely formally with reference to only very minimal claims about what these parameters might stand for. For example, the variation and interaction can be expressed in terms of set membership, where the only assumptions we need to make in order to secure the result that WOK and RKA are incompatible are that some of the members of these sets are not identical to each other and that there is a certain restriction on how set membership is determined (I will illustrate this with an example in $\S 3$ ). The assumption that some of the members are not identical to each other is philosophical in nature. In $\S 3$ it is uncontroversial; in $\S 4$ it is more controversial, but we will see that a persuasive defence is available that does not do any violence to one's broader commitments, for example, in the theory of perception. For this reason, I believe this assumption is minimal. The restriction on how set membership is determined is a direct consequence of relativist semantics and thus requires no defence here. It is because the variation and interaction can be understood purely formally, with minimal assumptions about what this formal system might stand for, that I say that WOK and RKA are incompatible as a consequence of the logic of RKA. I contrast this, especially in $\$ 5$, with more substantive philosophical commitments that might lead to the incompatibility of WOK and RKA. ${ }^{2}$

\footnotetext{
${ }^{1}$ I use 'knowledge attributions' to refer to both knowledge attributions proper and knowledge denials.

${ }^{2}$ I am grateful to an anonymous reviewer for pressing me to spell out this aspect of the dialectic.
} 
I will begin by demonstrating that it follows from the logic of what we might call local relativism, i.e. semantic relativism about knowledge attributions and no other sentences, that WOK is false. I will then argue that it follows from the logic of what we might call nonlocal relativism, i.e. semantic relativism about knowledge attributions and some other sentences, that WOK is false. To the best of my knowledge everyone who endorses relativism about knowledge attributions is a non-local relativist. However, it is easier to see that local relativism and WOK are incompatible than it is to see that non-local relativism and WOK are incompatible. It should also be easier to understand why non-local relativism and WOK are incompatible once we have examined local relativism.

Second, I argue that, even if WOK is incompatible with the main rivals to RKA, this is not a consequence of the logics of these views. Therefore, it is not open to proponents of RKA to dismiss my observations by pointing out that they apply equally to the main rivals of RKA.

Third, I consider some responses to the effect that it does not matter that WOK and RKA are incompatible. I conclude that they are implausible, do not fit our dialectic or require a lot more argument before they could be considered satisfactory.

There are several narrow and broader reasons why this paper should be interesting. On the narrow side, to the best of my knowledge, the relation between WOK and RKA has not been examined, so the discussion has intrinsic value. Furthermore, it highlights a hidden epistemological cost of RKA, which puts pressure on relativists to respond and to engage in more epistemology than they currently do. It also shows that RKA's main rivals do not have the same cost, so it contributes to the ongoing discussions about how to correctly describe the semantics of knowledge attributions (see, e.g., Tarasov 2013; 2014). On the broader side, this paper has some potentially serious consequences for the relativists' distinction between assessment-sensitive and assessment-insensitive sentences. I will return to these in the conclusion.

There are six further sections. In §2 I set out the general relativist framework, explain what it means to say that knowledge attributions are assessment-sensitive in terms of this framework and define logical implication. In $\S 3$ and $\S 4$ I show that both local and non-local relativism are incompatible with WOK. In $\$ 5$ I argue that, even if WOK is incompatible with the main rivals to RKA, this is not a consequence of the logics of these views. I consider moderate invariantism, attributor contextualism and subject-sensitive invariantism. In $§ 6$ I consider some ways of arguing that it does not matter that WOK and RKA are incompatible. I conclude in \$7. Appendix I accounts for, inter alia, important simplifications in my 
presentation of the relativist framework. It should serve to pre-empt any claims that I am working with an inaccurate characterisation of relativism. Appendix II demonstrates a point made in $\S 4$.

\section{Semantic relativism}

\subsection{General framework}

Setting aside some complications that are not relevant to our argument here (see Appendix I), we can sketch the relativist framework as follows. In order to determine the truth-value of a given sentence $\Phi$, the following must be established (MacFarlane 2014: Ch. 3 \& 4; cf. Kaplan 1989):

(i) The character of $\Phi$, defined as a function from the context of utterance of $\Phi$ to propositions.

(ii) The context of utterance of $\Phi$, defined as a possible world $w$ and understood to represent a possible occasion of use of $\Phi$.

(iii) The proposition expressed by $\Phi$, defined as a function from circumstances of evaluation to truth-values.

(iv) The circumstance of evaluation of the proposition expressed by $\Phi$, defined as a pair of the world $w_{c}$ of the context of utterance of $\Phi$ and an additional parameter $n_{a}$ determined by the context of assessment of $\Phi$, such that the values of $w_{c}$ and $n_{a}$ vary independently of each other.

(v) The context of assessment of $\Phi$, understood to represent a possible situation in which the use of $\Phi$ might be assessed.

We can use this framework to do three things: (i) make a distinction between assessment-sensitive and assessment-insensitive sentences; (ii) unpack RKA, i.e. say what exactly it is for knowledge attributions to be assessment-sensitive; and (iii) define the notion of logical implication, which we saw is at work in WOK. 


\subsection{Assessment-sensitivity and assessment-insensitivity}

The difference between assessment-sensitive and assessment-insensitive sentences can be explained as follows. Assessment-sensitive sentences express propositions that have different truth-values with respect to different values of the worlds of the contexts of utterance parameter $\mathrm{w}_{\mathrm{c}}$ or different values of the additional parameter $\mathrm{n}_{\mathrm{a}}$ determined by contexts of assessment of circumstances of evaluation. Assessment-insensitive sentences express propositions that have different truth-values with respect to different values of $\mathrm{w}_{\mathrm{c}}$ only (cf. MacFarlane 2014: 90). Formally speaking, the truth-values of propositions expressed by assessment-insensitive sentences must still be evaluated with respect to the value of $n_{a}$, but the value of $n_{a}$ makes no practical difference to the truth-value of these propositions.

$A S$ : A sentence $\Phi$ is assessment-sensitive if and only if, for all circumstances of evaluation that are pairs of a world of the context of utterance parameter $\mathrm{w}_{\mathrm{c}}$ and an additional parameter determined by contexts of assessment $\mathrm{n}_{\mathrm{a}}$, the proposition expressed by $\Phi$ is true with respect to one circumstance $\left\langle\mathrm{w}_{\mathrm{c} 1}, \mathrm{n}_{\mathrm{a} 1}\right\rangle$ and false with respect to another circumstance $\left\langle\mathrm{w}_{\mathrm{c} 1}, \mathrm{n}_{\mathrm{an}}\right\rangle$, where $\mathrm{n}_{\mathrm{an}}$ is a value other than $\mathrm{n}_{\mathrm{a} 1}$.

AIS: A sentence $\Phi$ is assessment-insensitive if and only if, for all circumstances of evaluation that are pairs of a world of the context of utterance parameter $w_{c}$ and an additional parameter determined by contexts of assessment $\mathrm{n}_{\mathrm{a}}$, the proposition expressed by $\Phi$ is true with respect to one circumstance $\left\langle\mathrm{w}_{\mathrm{c} 1}, \mathrm{n}_{\mathrm{a} 1}\right\rangle$ and true with respect to any other circumstance that is a pair of the world $\mathrm{w}_{\mathrm{c} 1}$ and any value of $\mathrm{n}_{\mathrm{a}}$.

The stock examples here are sentences like 'Orange juice is tasty' and 'Orange juice is acidic'. MacFarlane (see 2014: 2) would argue that the former sentence is assessmentsensitive, while the latter is assessment-insensitive. More precisely, he would replace the parameter $\mathrm{n}_{\mathrm{a}}$ in our two definitions above with a parameter for different standards of taste. He would then say that the proposition expressed by the sentence 'Orange juice is tasty' is true with respect to a world and one value of the standards of taste parameter and false with respect to the same world and another value of this parameter, where the values of this parameter are determined by different contexts of assessment. For instance, suppose that 
Larry likes orange juice, while Cheryl hates it. Then the proposition expressed by 'Orange juice is tasty' may be true with respect to a possible world and the standard of taste determined by Larry's context of assessment and false with respect to the same world and the standard determined by Cheryl's context of assessment. For short, we can say that the proposition expressed by 'Orange juice is tasty' is true relative to Larry and false relative to Cheryl. In contrast, the proposition expressed by the sentence 'Orange juice is acidic' is true with respect to a possible world and all standards of taste. For example, it is true relative to both Larry and Cheryl.

\subsection{Unpacking RKA}

In order to say that knowledge attributions are assessment-sensitive we need to replace the parameter $n_{a}$ in our definition of circumstances of evaluation in $\$ 2.1$ with a parameter for epistemic standards $e_{a}$, i.e. epistemic standards determined by contexts of assessment (MacFarlane 2005: 217-18; MacFarlane 2014: 188-89). Epistemic standards and the corresponding notion of epistemic positions should be understood in the usual way, i.e. as placeholders for our best theory of knowledge within a description of the semantics of knowledge attributions. Specifically, epistemic standards can stand in for any necessary or sufficient condition on knowledge other than truth and (confident) belief, and epistemic positions can stand in for the extent to which a given subject satisfies this condition (MacFarlane 2005: 217-18). For example, epistemic standards can stand in for different sets of alternatives to the target proposition, i.e. the proposition that is the object of knowledge, where an alternative is characterized as a proposition that is incompatible with the target proposition. The bigger the set of alternatives, the higher the epistemic standard; the smaller the set, the lower the standard. Correspondingly, epistemic positions can stand in for subjects' abilities to rule out alternatives. The more alternatives one is able to rule out, the stronger one's epistemic position; the fewer alternatives, the weaker the position. A subject's epistemic position must meet or exceed the relevant epistemic standard in order for the subject to satisfy the conditions on knowledge (cf. MacFarlane 2014: 177).

Following AS in $\$ 2.2$ above, we can now unpack RKA as follows:

$R K A^{*}$ : For all circumstances of evaluation that are pairs of a world of the context of utterance parameter $\mathrm{w}_{\mathrm{c}}$ and an epistemic standards parameter determined by contexts of assessment $\mathrm{e}_{\mathrm{a}}$, the proposition expressed by a knowledge attribution is true with 
respect to one circumstance $\left\langle\mathrm{w}_{\mathrm{c} 1}, \mathrm{e}_{\mathrm{a} 1}\right\rangle$ and false with respect to another circumstance $\left\langle\mathrm{w}_{\mathrm{cl}}, \mathrm{e}_{\mathrm{an}}\right\rangle$, where $\mathrm{e}_{\mathrm{an}}$ is a value other than $\mathrm{e}_{\mathrm{a} 1}$.

As we might expect then, relativism about knowledge attributions works in a similar way to relativism about sentences like 'Orange juice is tasty'. Take the sentence 'Alice knows that Humpty Dumpty had a fall'. Suppose that Humpty Dumpty did have a fall, Alice (confidently) believes that he did and has some justification for believing this. Suppose as well that Larry's context of assessment sets a very high epistemic standard, while Cheryl's sets a very low epistemic standard. Then the proposition expressed by 'Alice knows that Humpty Dumpty had a fall' might be true relative to Cheryl and false relative to Larry.

\subsection{Logical implication}

MacFarlane (2014: 68) defines logical implication with respect to sentences and open formulas, not propositions. However, what MacFarlane says about sentences extends mutatis mutandis to propositions (see Appendix I). Given this, and the fact that we are treating circumstances of evaluation as pairs of worlds of the contexts of utterance and an additional parameter determined by contexts of assessment (see §2.1), we can extend MacFarlane’s definition of logical implication to propositions as follows:

Logical implication: A proposition $\mathrm{p}$ is logically implied by a set $\mathrm{Q}$ of propositions if and only if for every circumstance of evaluation $j$, if every member of $Q$ is true at $j$, then $p$ is true at $j$.

We can now show that WOK and RKA are incompatible.

\section{Local relativism and ways of knowing}

Local relativism is the view that knowledge attributions and no other sentences are assessment-sensitive. Non-local relativism is the view that knowledge attributions and some other sentences are assessment-sensitive. As noted in $\S 1$, the dialectic is easier to follow if we examine local relativism before we turn to non-local relativism.

Consider the following two sentences: 
(1) Alice sees that Humpty Dumpty had a fall.

(2) Alice knows that Humpty Dumpty had a fall.

Assume that there are no lexically or structurally ambiguous, no indexical and no hidden syntactic elements in (1) and (2). Given this, relativists would accept that (1) and (2) express the following propositions respectively:

ALICE $_{1}$ : that Alice sees that Humpty Dumpty had a fall.

$\mathrm{ALICE}_{2}$ : that Alice knows that Humpty Dumpty had a fall.

Following local relativism, (1) is assessment-insensitive and (2) assessmentsensitive. Since (1) and (2) are the only sentences we are concerned with here, we do not need to make any alterations to the relativist framework sketched in $\S 2$. That is, we can take circumstances of evaluation to be pairs of worlds $w_{c}$ of the contexts of utterance and epistemic standards $e_{a}$ determined by contexts of assessment: $\left\langle w_{c}, e_{a}\right\rangle$. Then the assessmentinsensitivity of (1) amounts to this: for all circumstances of evaluation that are pairs of $\mathrm{w}_{\mathrm{c}}$ and $e_{a}, A_{L I C E}$ is true with respect to one circumstance $\left\langle w_{c 1}, e_{a 1}\right\rangle$ and true with respect to any other circumstance that is a pair of the world $\mathrm{w}_{\mathrm{c} 1}$ and any value of $\mathrm{e}_{\mathrm{a}}$. The assessmentsensitivity of (2) amounts to this: for all circumstances that are pairs of $w_{c}$ and $e_{a}, A_{L I C E}$ is true with respect to one circumstance $\left\langle\mathrm{w}_{\mathrm{c} 1}, \mathrm{e}_{\mathrm{a} 1}\right\rangle$ and false with respect to another circumstance $\left\langle w_{c 1}, e_{a n}\right\rangle$, where $e_{a n}$ is a value other than $e_{a 1}$. But now we can see that there must be at least one circumstance of evaluation at which $\mathrm{ALICE}_{1}$ is true but $\mathrm{ALICE}_{2}$ is false. For example, suppose that $\mathrm{ALICE}_{1}$ is true at the following circumstances of evaluation: $\left\langle w_{c 1}, e_{a 1}\right\rangle,\left\langle w_{c 1}, e_{a}\right\rangle$ and so on for every circumstance of evaluation that is a pair of the value $\mathrm{w}_{\mathrm{c} 1}$ and any value of $\mathrm{e}_{\mathrm{a}}$. We must suppose that $\mathrm{ALICE}_{2}$ is false at at least one of these circumstances of evaluation; it is entailed by the claim that (2) is assessment-sensitive that there is at least one epistemic standard with respect to which ALICE $_{2}$ is false, and therefore there is at least one circumstance of evaluation that is a pair of the value $w_{c 1}$ and a value of $e_{a}$ at which $\mathrm{ALICE}_{2}$ is false.

To clarify what is going on here, note that MacFarlane sometimes (e.g. 2014: 76-81) introduces the notion of assessment-sensitivity by drawing a direct analogy between 
assessment-sensitive sentences and contingently true or contingently false sentences. In a possible worlds semantic model for contingent sentences, there must be at least one possible world at which the proposition expressed by a contingent sentence is true and at least one possible world at which the proposition is false. Otherwise, the sentence would not be contingently true or contingently false; it would be necessarily true or necessarily false. Likewise, in a semantic model for assessment-sensitive sentences, there must be at least one value of a parameter fixed by a context of assessment with respect to which the proposition expressed by an assessment-sensitive sentence is true and at least one value of this parameter with respect to which the proposition is false. Otherwise, the sentence would not be assessment-sensitive; it would be assessment-insensitive. If we now include both possible worlds and a parameter determined by contexts of assessment in a single semantic model - the kind of model MacFarlane explicitly has in mind and we are working with here then in this semantic model the truth-value of the proposition expressed by a contingently true or false assessment-sensitive sentence must vary across possible worlds and values of a parameter determined by contexts of assessment.

To see this better, let us assume sentences (1) and (2) are contingently true and imagine that we are working in a toy semantic model that has four, and only four, circumstances of evaluation: $\left\langle w_{c 1}, e_{a 1}\right\rangle,\left\langle w_{c 1}, e_{a 2}\right\rangle,\left\langle w_{c 2}, e_{a 1}\right\rangle$ and $\left\langle w_{c 2}, e_{a 2}\right\rangle$. Given that sentence (2) is contingently true and assessment-sensitive, suppose that $\mathrm{ALICE}_{2}$ is true at $\left\langle w_{c 1}, e_{a 1}\right\rangle$. Then ALICE $E_{2}$ must be false at every other circumstance of evaluation in our toy model. Given that sentence (1) is contingently true and assessment-insensitive, suppose that $\mathrm{ALICE}_{1}$ is true at $\left\langle\mathrm{w}_{\mathrm{c} 1}, \mathrm{e}_{\mathrm{a} 1}\right\rangle$. Then $\mathrm{ALICE}_{1}$ must also be true at $\left\langle\mathrm{w}_{\mathrm{c} 1}, \mathrm{e}_{\mathrm{a} 2}\right\rangle$ and false at every other circumstance of evaluation in our toy model. But then there is one circumstance of evaluation, viz. $\left\langle\mathrm{w}_{\mathrm{c} 1}, \mathrm{e}_{\mathrm{a} 2}\right\rangle$, in our toy model at which $\mathrm{ALICE}_{1}$ is true but $\mathrm{ALICE}_{2}$ is false, as shown in Table 1 below. $^{3}$

\footnotetext{
${ }^{3}$ For a given circumstance of evaluation, read down from the worlds columns (' $\mathrm{w}_{\mathrm{c} 1}$ ', ' $\mathrm{w}_{\mathrm{c} 2}$ ') and across from the epistemic standards rows (' $\mathrm{e}_{\mathrm{a} 1}$ ', ' $\mathrm{e}_{\mathrm{a} 2}$ '); the circumstances at which $\mathrm{ALICE}_{1}$ and $\mathrm{ALICE}_{2}$ are true are marked with 'ALICE 1 ' and 'ALICE' 2 respectively.
} 
Table 1: Circumstances of evaluation at which $A L I C E_{1}$ and $A L I C E_{2}$ are true and circumstances at which they are false in the local relativism example

\begin{tabular}{|c|c|c|}
\cline { 2 - 3 } \multicolumn{1}{c|}{} & $\mathbf{w}_{\mathbf{c}}$ & \multirow{2}{*}{$\mathbf{w}_{\mathbf{c} 2}$} \\
\hline $\mathbf{e}_{\mathbf{a} 1}$ & $\mathrm{ALICE}_{1}$ & \\
& $\mathrm{ALICE}_{2}$ & \\
\hline $\mathbf{e}_{\mathbf{a} 2}$ & $\mathrm{ALICE}_{1}$ & \\
\end{tabular}

Now, following the definition of logical implication in $\S 2.4$, to say that there is at least one circumstance of evaluation at which $\mathrm{ALICE}_{1}$ is true but $\mathrm{ALICE}_{2}$ is false is just to say that $\mathrm{ALICE}_{1}$ does not logically imply $\mathrm{ALICE}_{2}$. Of course, according to WOK, ALICE 1 does logically imply ALICE 2 . So it follows from the logic of local relativism that WOK is false. $^{4}$

\footnotetext{
${ }^{4}$ I am grateful to an anonymous reviewer for pointing out that there could be an epistemic standard that is so high that if one knows that $\mathrm{p}$ relative to that epistemic standard, then one knows that $\mathrm{p}$ relative to any epistemic standard, i.e. one could be in the strongest possible epistemic position with respect to p. For instance, Alice could be in the strongest possible epistemic position with respect to the proposition that Humpty Dumpty had a fall. Here it would not be the case that there must be a circumstance of evaluation at which ALICE 1 is true but $\mathrm{ALICE}_{2}$ is false. Taking our toy model from the main text for demonstration, ALICE 2 could be true at both $\left\langle\mathrm{w}_{\mathrm{c} 1}, \mathrm{e}_{\mathrm{a} 1}\right\rangle$ and $\left\langle\mathrm{w}_{\mathrm{c} 1}, \mathrm{e}_{\mathrm{a} 2}\right\rangle$, since Alice's epistemic position could meet both standards $e_{\mathrm{a} 1}$ and $\mathrm{e}_{\mathrm{a} 2}$. However, recall that what is at stake here is the compatibility of WOK and RKA, so in order to make this response work for RKA one has to suppose that for any pair of propositions expressed by a perception sentence and a knowledge attribution, like the propositions $\mathrm{ALICE}_{1}$ and $\mathrm{ALICE}_{2}$, the subject of these sentences is in the strongest possible epistemic position with respect to the object of these sentences. In other words, one has to commit to what we can call perception infallibilism (PI): for any subject $\mathrm{x}$ and any proposition $\mathrm{p}$, that $\mathrm{x}$ perceives that $\mathrm{p}$ puts $\mathrm{x}$ in the strongest possible epistemic position with respect to $\mathrm{p}$. PI is controversial and obviously not part of WOK or RKA. Of course, there may well be good reasons to believe that PI is true, but the onus would be on relativists to give these reasons. Moreover, we will see in $\S 5$ that the main rivals to RKA do not have the same issues with WOK, so it follows that they do not need to appeal to PI. Therefore, even if relativists could give good reasons for accepting PI, RKA would still come with some controversial philosophical consequences that its main rivals do not.
} 
This way of modelling our example should help to illustrate what I meant in $§ 1$ when I said that the variation in the values of parameters and interaction between parameters can be expressed purely formally, for example in terms of set membership, where the only assumptions we need to make in order to secure the result that WOK and RKA are incompatible are that the members of the relevant sets are not identical to each other and that there is a certain restriction on how set membership is determined. In the present case, $\mathrm{ALICE}_{1}$ logically implies ALICE 2 only if the set of circumstances at which $\mathrm{ALICE}_{1}$ is true is a subset of the set of circumstances at which $\mathrm{ALICE}_{2}$ is true. Looking at Table 1, this means that $\left\{\left\langle w_{c 1}, e_{a 1}\right\rangle,\left\langle w_{c 1}, e_{a 2}\right\rangle\right\}$ would need to be a subset of $\left\langle w_{c 1}, e_{a 2}\right\rangle$, which, of course, it is not. The assumption that the members of these sets are not identical here translates to the assumption that, for any values of parameters $w_{c}$ and $e_{a}, w_{c}$ is not identical with $e_{a}$, which we have worked with implicitly. And the restriction on how set membership is determined here translates to how the distinction between assessment-sensitive and assessment-insensitive sentences works to generate the set of circumstances $\left\{\left\langle w_{c 1}, e_{a 1}\right\rangle,\left\langle w_{c 1}, e_{a 2}\right\rangle\right\}$ with respect to $\mathrm{ALICE}_{1}$ and the set of circumstances $\left\langle\mathrm{w}_{\mathrm{c} 1}, \mathrm{e}_{\mathrm{a} 2}>\right.$ with respect to ALICE 2 . Now, crucially, notice that no matter what claims we make about the things $\mathrm{w}_{\mathrm{c} 1}, \mathrm{e}_{\mathrm{a} 1}$ and $\mathrm{e}_{\mathrm{a} 2}$ stand for, it remains the case that $\left\{\left\langle w_{c 1}, e_{a 1}\right\rangle,\left\langle w_{c 1}, e_{a 2}\right\rangle\right\}$ is not a subset of $\left\langle w_{c 1}, e_{a 2}\right\rangle$. It is in this sense that the variation and interaction between parameters can be expressed purely formally; and in this sense it is a consequence of the logic of RKA that WOK and RKA are incompatible. As we will see in $§ 5$, this is different for rivals to RKA, where, in order to render these rivals incompatible with WOK, more substantial philosophical assumptions would need to be made about what parameters like $\mathrm{e}_{\mathrm{a}}$ stand for and how this fits within a broader epistemology.

Put less formally, the reason why $\mathrm{ALICE}_{1}$ does not logically imply $\mathrm{ALICE}_{2}$ here is that the truth-value of $\mathrm{ALICE}_{2}$ varies across different parameters to the truth-value of $\mathrm{ALICE}_{1}$ and the values of these parameters vary independently of each other. We can sum this up by saying that the truth-values of $\mathrm{ALICE}_{1}$ and $\mathrm{ALICE}_{2}$ do not co-vary in the right way. More generally then, we can say that local relativism and WOK are incompatible because the truth-values of the proposition expressed by a perception sentence and the proposition expressed by a knowledge attribution do not co-vary in the right way. ${ }^{5}$

\footnotetext{
${ }^{5}$ We have defined WOK in terms of logical implication, but one might wonder if WOK could be defined in terms of an alternative notion and whether the present argument would still go through in that case. For example, WOK*: for any subject $\mathrm{x}$ and sentence $\Phi,\ulcorner\mathrm{x}$ knows that $\Phi\urcorner$ is a logical consequence of $\ulcorner\mathrm{x}$ perceives that $\Phi\urcorner$, where logical consequence is defined as preservation of truth at a context of utterance and a context of assessment. Firstly, note that WOK* works with sentences rather than propositions. We might wonder whether
} 


\section{Non-local relativism and ways of knowing}

Non-local relativists believe that knowledge attributions and some other sentences are assessment-sensitive. In the present context, it is easy to imagine that they would endorse the claim that both perception sentences and knowledge attributions are assessmentsensitive. We need to establish whether this claim is compatible with WOK. On the face of it, we might think that it is. In the previous section we saw that local relativism and WOK are incompatible because the truth-values of the proposition expressed by a perception sentence and the proposition expressed by a knowledge attribution do not co-vary in the right way. If we now say that both perception sentences and knowledge attributions are assessment-sensitive, we might expect the truth-values of the propositions expressed by these sentences to co-vary in the right way, and therefore for non-local relativism to be compatible with WOK. In fact, it turns out there are good reasons to think that this is not the case. In particular, there are good reasons to think that the truth-values of these propositions vary with respect to different parameters, where the values of these parameters vary independently of each other. This should become clear once we consider what might be involved in the claim that perception sentences are assessment-sensitive.

We have seen that, following RKA, the proposition expressed by a knowledge attribution is sensitive to changes in epistemic standards determined by contexts of assessment. Now, it seems to me clearly implausible that the claim that perception sentences are assessment-sensitive should amount to the same thing, i.e. that the proposition expressed by a perception sentence is sensitive to changes in epistemic standards determined by contexts of assessment. Why? Recall that epistemic standards and the corresponding notion of epistemic positions are placeholders for our best theory of knowledge (see $\S 2.3$ ). In light

this gets to the heart of the view that perceiving entails knowing. Specifically, we might want to say that, unlike $\mathrm{WOK}^{*}$, this view is about more than just a relation between perception sentences and knowledge attributions. Secondly, our argument still goes through with WOK*. Recall that circumstances of evaluation are defined as pairs of the world of the context of utterance and an epistemic standard set by a context of assessment. Suppose for sentences (1) and (2) the context of utterance is $c_{1}$ and the context of assessment is $a_{2}$. By analogy with our toy semantic model above (see Table 1), it is possible for sentence (1) to be true at $c_{1}$ and $a_{2}$ and for sentence (2) to be false at $c_{1}$ and $a_{2}$, in which case truth is not preserved at a context of utterance and a context of assessment and therefore (2) is not a logical consequence of (1). Thanks to an anonymous referee for pressing this point and for defining $\mathrm{WOK}^{*}$. 
of this, it is plausible to view perception as something that determines the strength of a subject's epistemic position and thus the extent to which the subject meets an epistemic standard; it is then implausible to view perception as something that is itself relative to an epistemic standard. For instance, suppose that $\mathrm{ALICE}_{1}$ is true, i.e. it is true that Alice sees that Humpty Dumpty had a fall. That Alice sees that Humpty Dumpty had a fall puts Alice in a certain epistemic position (following WOK, an epistemic position sufficient for knowledge) with respect to the proposition that Humpty Dumpty had a fall. It is then implausible that that Alice sees that Humpty Dumpty had a fall is itself relative to an epistemic standard.

One might object to this reasoning on at least three grounds. Firstly, one might think that it is not at all, or at least not clearly, implausible that that the proposition expressed by a perception sentence is sensitive to changes in epistemic standards. For example, suppose that epistemic standards stand for the ability to rule out different sets of alternatives to the target proposition, where an alternative is characterized as a proposition that is incompatible with the target proposition (cf. §2.3). For example, assuming that Humpty Dumpty is not identical with Pickle Rick (an anthropomorphised pickle) and that only one of them had a fall, the proposition that Pickle Rick had a fall is an alternative to the proposition that Humpty Dumpty had a fall. Then one might say that Alice sees that Humpty Dumpty had a fall only if she can rule out, say, that Pickle Rick had a fall. This seems perfectly acceptable, so we have at least prima facie evidence that $\mathrm{ALICE}_{1}$ is sensitive to changes in epistemic standards.

However, notice that this objection leaves no room for the plausible claim that perception is something that determines the strength of a subject's epistemic position and thus the extent to which the subject meets an epistemic standard. This is a weaker claim than WOK. One can believe that, for any subject $\mathrm{x}$ and any proposition $\mathrm{p}$, that $\mathrm{x}$ perceives that $\mathrm{p}$ puts $\mathrm{x}$ in a certain epistemic position with respect to $\mathrm{p}$, but not believe that this epistemic position is sufficient to know that $\mathrm{p}$. Indeed, I think it is fair to say that the weaker claim is almost platitudinous; even global sceptics should have no trouble accepting it (see, e.g., Williams 1996: Ch. 2).

To see that the present objection leaves no room for this claim, suppose that Alice knows that Humpty Dumpty had a fall only if she can rule out that Pickle Rick had a fall. One way in which Alice can rule out that Pickle Rick had a fall is by seeing that Humpty Dumpty had a fall (obviously assuming only one object had a fall). However, according to the present objection, Alice sees that Humpty Dumpty had a fall only if she can rule out that Pickle Rick had a fall. Whatever it is that allows Alice to rule out that Pickle Rick had a fall cannot itself depend on Alice ruling out that Pickle Rick had a fall, else it 
would be of no use to Alice as a means of ruling out that Pickle Rick had a fall. To reinforce this point, consider the following conversation:

Cheryl: Alice knows that Humpty Dumpty had a fall.

Larry: She doesn't know it if she can't rule out it was Humpty Dumpty and not Pickle Rick.

Cheryl: Well, she saw it was Humpty Dumpty.

Larry: She didn't see it if she can't rule out it was Humpty Dumpty and not Pickle Rick.

Larry's first utterance makes salient the alternative that needs to be ruled out, viz. that Pickle Rick had a fall. In making her second utterance, Cheryl presupposes that Alice can rule out that Pickle Rick had a fall by seeing that Humpty Dumpty had a fall. And Larry's second utterance makes the claim that Alice saw that Humpty Dumpty had a fall only if she can rule out that Pickle Rick had a fall. If both perception sentences and knowledge attributions are sensitive to changes in epistemic standards - here, changes in relevant alternatives - and if perception can determines the strength of a subject's epistemic position, then Larry's second utterance should be perfectly acceptable. Yet I do not think it is. It seems to me that Larry is either questioning whether seeing that $\mathrm{p}$ really supplies adequate evidence for the truth of $\mathrm{p}$, or he is doing no more than obstinately contradicting Cheryl. Indeed, the most natural response to Larry seems to be something like 'I just told you; she saw it was Humpty Dumpty'. Thus, if we want to maintain that both perception sentences and knowledge attributions are sensitive to changes in epistemic standard, we have to abandon the claim that perception can determine the strength of a subject's epistemic position. ${ }^{6}$

\footnotetext{
${ }^{6}$ We might think that there are facts about perception, for example low-level facts about a subject's subpersonal perceptual processing, that determine the strength of a subject's epistemic position, and these facts can be expressed by assessment-insensitive sentences (I am grateful to a reviewer for this suggestion and wording). However, notice that if we follow this suggestion through, then that one is in a certain perceptual process should logically imply that one perceives something. For instance, that Alice is in a certain perceptual process (call this proposition ' $\mathrm{ALICE}_{3}$ ') should logically imply that Alice perceives something; $\mathrm{ALICE}_{3}$ should logically imply $\mathrm{ALICE}_{1}$. Now, just as $\mathrm{ALICE}_{1}$ is expressed by sentence (1), so presumably ALICE 3 is expressed by (3): 'Alice is in a certain perceptual process'. But if we hold that (3) is assessment-insensitive and (1) is assessmentsensitive, then we get the exact analogue of the problem discussed in $\S 3$, viz. ALICE 3 does not logically imply
} 
Alternatively, one might argue that the claim that it is implausible that the proposition expressed by a perception sentence is sensitive to changes in epistemic standards ignores the wider discussion of perception, where it might be thought that the proposition expressed by a perception sentence is sensitive to changes in epistemic standards. In response, recall that epistemic standards are placeholders; in our examples they have stood in for varying abilities to rule out alternatives, but they could stand in for something else: evidence for the target proposition, the ability to track the truth of a proposition or whatever else. As such, when someone says that the proposition expressed by a perception sentence is sensitive to changes in epistemic standards, what they mean is that that proposition is sensitive to, for example, varying abilities to rule out alternatives. But there is no principled reason to treat epistemic standards as the placeholder for this sort of thing; other placeholders could be introduced. For instance, we could say that the proposition expressed by a perception sentence is sensitive to changes in visual standards (whatever they might be), and we could say that visual standards stand in for varying abilities to rule out alternatives. In short, the thought that the proposition expressed by a perception sentence is sensitive to changes in epistemic standards is really the thought that that proposition is sensitive to changes in some relevant notion, which for convenience we represent with epistemic standards, but could represent with another placeholder. Thus, we can reconcile the claim that it is implausible that the proposition expressed by a perception sentence is sensitive to changes in epistemic standards with whatever else we might want to say about perception. Moreover, since the proposition expressed by a knowledge attribution is sensitive to changes in epistemic standards and the proposition expressed by a perception sentence is sensitive to another placeholder, notice that in the logic of RKA we end up with two distinct parameters which vary independently of each other, which is just what we want here.

Finally, one might suggest that, if WOK and RKA are both true and my reasoning in $\$ 3$ is correct, it is entailed that both perception sentences and knowledge attributions are sensitive to changes in epistemic standards determined by contexts of assessment. However, this begs the question against the point at issue; although here we do allow the claim that perception sentences are assessment-sensitive, we dispute that this claim should be cashed out as the idea that perception sentences are sensitive to changes in epistemic standards determined by contexts of assessment. In other words, one could claim that, if WOK and imply that one perceives something (cf. $\$ 7$ and Tarasov 2014: 177-78). 
RKA are both true and $\S 3$ is sound, it is entailed that both perception sentences and knowledge attributions are assessment-sensitive. However, one could not claim on the same basis that these sentences are assessment-sensitive in the same way, i.e. it is not entailed that they are both sensitive to changes in epistemic standards; this latter point requires independent support. This is reinforced by the fact that there are good independent reasons, e.g. those set out in the context of the Pickle Rick examples above, to think that if perception sentences are assessment-sensitive, they are sensitive to a parameter other than epistemic standards.

Given our reasoning then, the claim that perception sentences are assessmentsensitive amounts to the claim that the proposition expressed by a perception sentence is sensitive to changes in some factor, determined by contexts of assessment, other than epistemic standards. It follows that the truth-values of the propositions expressed by a knowledge attribution and a perception sentence vary with respect to different parameters determined by contexts of assessment, where the values of these parameters vary independently of each other. Given what we learnt in $\S 3$, we should expect that the truthvalues of these propositions do not co-vary in the right way. I demonstrate that this expectation is correct in Appendix II, but, to move things along, here I conclude that nonlocal relativism and WOK are incompatible. ${ }^{7}$

\footnotetext{
${ }^{7}$ I am grateful to a reviewer for querying whether we cannot be convinced the truth-values of these propositions might co-vary in the right way after all. They have in mind Dretske (1970: 1015-16) and Goldman (1976: 77273) style scenarios in which a subject $\mathrm{x}$ appears to perceive zebras or barns, but they turn out to be cleverly painted mules or barn facades. The suggestion is that in such cases we would say that $\mathrm{x}$ only appears to perceive zebras or barns, but does not really, just as we would say that x only appears to know there are zebras or barns in front of $\mathrm{x}$, but does not really. This seems to show that we are willing to retract an utterance of a perception sentence just when we are we willing to retract an utterance of a knowledge attribution, which in turn might show that the truth-values of the propositions expressed by these sentences co-vary in the right way, and in particular that the truth-values of the propositions expressed by knowledge attributions and perception sentences are both sensitive to changes in epistemic standards. In fact, I think these examples work in our favour. Firstly, they lend indirect support to WOK; we would not bother retracting an utterance of a knowledge attribution that was based on an utterance of a perception sentence if we did not think that perception was sufficient for knowledge in the first place. Secondly, the examples only work if we assume that the only basis for the claim that $\mathrm{x}$ knows that there are zebras or barns is that $\mathrm{x}$ perceives that there are. But often the basis for knowing something is non-perceptual, e.g. deductive or testimonial, or a combination of perceptual and nonperceptual. Thus, we can argue that whatever constitutes x's epistemic position and, as a corollary, an epistemic standard, is richer than whatever might constitute a standard for perception (if there is such a thing). As a result, the truth-values of the propositions expressed by perception sentences and knowledge attributions are not both
} 


\section{Rivals to RKA and ways of knowing}

It is a popular strategy to dismiss an observation by pointing out that it also applies to one's rivals. Thus, one might attempt to dismiss the observations in the previous two sections by claiming that WOK is incompatible with the rivals to RKA as a consequence of their logics. Notice that it would not be enough to claim simply that WOK is incompatible with the rivals to RKA. A key point here is that the incompatibility of WOK and RKA is $a$ consequence of the logic of RKA. And one would struggle to show that any incompatibility between WOK and these rivals is a consequence of their logics. To see this, let us first consider moderate invariantism about knowledge attributions, one of the main rivals to RKA.

According to moderate invariantism, a knowledge attribution $\ulcorner\mathrm{x}$ knows that $\Phi\urcorner$ is true only if the epistemic position of the subject $\mathrm{x}$ meets a moderately high epistemic standard with respect to the proposition expressed by $\Phi$ (see, e.g., Brown 2006: 42428). Unlike semantic relativists, moderate invariantists do not believe that the epistemic standard varies across contexts of assessment. Indeed, moderate invariantists do not believe the epistemic standard varies at all; a single moderately high epistemic standard characterises the semantics of all knowledge attributions. Therefore, moderate invariantists do not need to include an epistemic standards parameter in their characterisation of circumstances of evaluation. In light of this, and given our reasoning in the previous two sections, we should not expect any incompatibility between WOK and moderate invariantism to be a consequence of the logic of moderate invariantism. For example, moderate invariantists could characterise circumstances of evaluation simply as sets of the worlds of the contexts of utterance $w_{c}$. In this case, we would not have enough parameters in circumstances to get the kind of interaction between the values of the parameters that we saw in $\S 3$.

If it does turn out that WOK and moderate invariantism are incompatible, we will find that this is a consequence of some substantive claim about the nature of perception or epistemic standards, not a consequence of the logic of moderate invariantism. For example, we might find that seeing that $\mathrm{p}$ puts one in a certain epistemic position with respect to $\mathrm{p}$, but that this epistemic position is not strong enough to meet a moderately high epistemic standard

sensitive to an epistemic standard. Lastly, recall that these examples are cases where $\mathrm{x}$ appears to perceive, but does not perceive. Although they might be suggestive of what happens in cases where $\mathrm{x}$ actually perceives, they do not say anything directly about these cases. 
(cf. §4). On this view, WOK and moderate invariantism would be incompatible, but this would be a consequence of a substantive claim about perception, not of the moderate invariantist characterisation of circumstances of evaluation, and hence not a consequence of the logic of moderate invariantism.

Similar arguments can be made for attributor contextualism and subject sensitive invariantism (SSI), the other major rivals to RKA. The difference between SSI and moderate invariantists is that, unlike the latter, subject sensitive invariantists (SSI-ists) believe that a subject's epistemic position is affected by their practical interests. In particular, SSI-ists believe that, all other things being equal, the more that is practically at stake for a subject, the weaker their epistemic position. Conversely, the less that is practically at stake for a subject, the stronger their epistemic position. However, SSI-ists can give the same logic for knowledge attributions as moderate invariantists. In particular, SSI-ists do not need to include an epistemic standards parameter in their characterisation of circumstances of evaluation and are free to characterise circumstances simply as sets of the worlds of the contexts of utterance (cf. Hawthorne 2004: 158). Given what we just saw about moderate invariantism, it is plain that, if SSI is incompatible with WOK, this is not a consequence of its logic.

According to attributor contextualism, a knowledge attribution $\ulcorner\mathrm{x}$ knows that $\Phi\urcorner$ is true only if the epistemic position of the subject $\mathrm{x}$ meets the relevant epistemic standard with respect to the proposition expressed by $\Phi$, where the relevant epistemic standard is determined by the context of utterance of the knowledge attribution (cf. DeRose 2009: 4766). Importantly, as a consequence of the variation in epistemic standards, the knowledge attribution may express different propositions in different contexts of utterance. There are thus two very important differences between attributor contextualism and relativism. First, whereas relativists believe that sentence (2) - 'Alice knows that Humpty Dumpty had a fall' - expresses the proposition $\mathrm{ALICE}_{2}$, attributor contextualists believe that sentence (2) may express not only $\mathrm{ALICE}_{2}$, but many other propositions as well. Second, whereas relativists build a parameter for epistemic standards into their definition of circumstances of evaluation, attributor contextualists build it into their definition of contexts of utterance. As a result, attributor contextualists do not need to include an epistemic standards parameter in their characterisation of circumstances of evaluation and are free to characterise circumstances simply as sets of the worlds of the contexts of utterance. This much is analogous with the logic of moderate invariantism Thus, we should see that if attributor contextualism is incompatible with WOK, this is not a consequence of its logic. For example, it might turn 
out that $\mathrm{ALICE}_{1}$ does not logically imply some of the propositions that, according to attributor contextualism, might be expressed by (2). However, it does not follow that ALICE $_{1}$ does not logically imply other propositions that might be expressed by (2). Therefore, whether attributor contextualism and WOK are incompatible is a moot point.

To sum up, in $\S 1$ we noted that the variation in the values of and interaction between parameters of circumstances of evaluation can be understood purely formally, with reference to only very minimal claims about what these parameters might stand for. We illustrated this in $\S 3$ by showing what happens when parameters $w_{c}$ and $e_{a}$ interact in a toy semantic model and by arguing that we need only very minimal claims about these parameters before WOK and RKA become incompatible. What we now find is that a similar move is not available in the case of RKA's rivals. None of these rivals feature a parameter $e_{a}$, or its analogue, in circumstances of evaluation. Thus, there is no relevant interaction between parameters of circumstances and therefore no parallel to the issue we have found for RKA. ${ }^{8}$

\section{It matters that RKA and WOK are incompatible}

Besides the argument considered in the previous section, there are three general types of response one could try to make to the observation that WOK and RKA are incompatible. First, WOK is not philosophically significant and therefore it does not matter that WOK and RKA are incompatible. Second, even if WOK is philosophically significant, relativists' approach to natural language semantics permits them to ignore it. Third, even if WOK is philosophically significant, there are good reasons to think it is false, so it is not a problem for RKA that it is incompatible with WOK.

We have seen that WOK enjoys a long philosophical pedigree, so the least we can say about it is that it is philosophically significant. Therefore, we can dismiss the first type of response out of hand.

The thought that relativists' approach to natural language semantics permits them to ignore the observation that WOK and RKA are incompatible has been suggested to me on the basis of the following reasoning. RKA is meant to describe the semantics of a part of natural language. We might think that the evidence for the semantics of natural language comes from ordinary speakers' intuitions and perhaps their beliefs about utterances of sentences of natural language. We might then think that the incompatibility of WOK and RKA matters

\footnotetext{
${ }^{8}$ For a detailed comparison of the semantics of the views discussed here, see Tarasov 2014.
} 
only if it implies that ordinary speakers' intuitions or beliefs are in error. And we could try to develop the thought that the incompatibility of WOK and RKA need not imply that ordinary speakers' intuitions or beliefs are in error. For example, we could follow Niko Kolodny and MacFarlane's (2010) or Tamina Stephenson (2007) and adopt a relativist semantics for indicative conditionals, i.e. sentences of the form $\ulcorner$ If $\Phi$, then $\Psi\urcorner$. We might then find that this semantics respects ordinary speakers' intuitions about sentences like 'If Alice sees that Humpty Dumpty had a fall, then Alice knows that Humpty Dumpty had a fall', and we could argue that these sentences look very much like instances of WOK.

There are several problems with this type of response. First, it is an obvious additional cost to RKA; we find that in order to keep WOK, a relativist about knowledge attributions also needs to become a relativist about indicative conditionals (cf. Tarasov 2014: 172-77). Second, recall that WOK concerns a relation of logical implication between propositions, not a relation between sentences or sentential clauses. It is notoriously controversial whether the relation of logical implication can be expressed in terms of indicative conditionals (e.g. Edgington 1995), and thus it is controversial whether the present approach really speaks to WOK (cf. fn. 5). Finally, notice that if one does not care that WOK and RKA are incompatible because RKA need not imply that ordinary speakers' intuitions or beliefs are in error, one must think that ordinary speakers' intuitions and beliefs are the only, or at least the most important, evidence for the semantics of knowledge attributions. But this attitude is extremist and rules out other popular considerations - for example, the theoretical virtues of a view, such as simplicity, consistency and so on - as evidence for the semantics of natural language (see, e.g., Daly and Liggins 2011).

Finally, consider the third type of response to the observation that WOK and RKA are incompatible, viz. the suggestion that this claim does not matter because WOK is false. There are two ways to develop this suggestion. We could assume that RKA is true, perhaps on the basis of independent evidence (see MacFarlane 2005: 218-19; MacFarlane 2014: 18790), and then reason from this and the premise that WOK is incompatible with RKA to the conclusion that WOK is false. The reasoning is an obvious instance of modus tollens, so it is valid. However, it does not fit the present dialectic, since what is at issue in this section is whether the incompatibility of WOK and RKA is a cost to RKA, thus one cannot dismiss WOK on the grounds that it is incompatible with RKA. A better way to develop this response is to show that there are independent reasons to think that WOK is false (see, e.g., Turri 2010). It is beyond the scope of this paper to examine these reasons, but since we are not aiming to refute RKA here - merely to note a hidden cost of the view and compare this to 
its closest rivals - we do not need to. We should have already done enough to shift the onus on relativists to pick up the argument here.

\section{Conclusion}

I have focused exclusively on WOK and RKA in this paper, but there are interesting questions about its broader relevance. ${ }^{9}$ Firstly, I believe the observations about WOK and RKA generalise. Recall that, in the demonstration above, the reason why ALICE 1 does not logically imply $\mathrm{ALICE}_{2}$ is that the truth-value of $\mathrm{ALICE}_{2}$ varies across different parameters to the truth-value of $\mathrm{ALICE}_{1}$ and the values of these parameters vary independently of each other. This demonstration generalises to other propositions and parameters, provided the basic features of the interaction between these parameters remain constant. That is to say, if one takes any pair of an assessment-insensitive and an assessment-sensitive sentence, one will find that the proposition expressed by the former does not logically imply the proposition expressed by the latter. Likewise, if one takes any pair of assessment-sensitive sentences, one will find the proposition expressed by one sentence will not logically imply the proposition expressed by the other, provided the truth-values of the two propositions vary with respect to different parameters, where the values of these parameters vary independently of each other. I do not have space to prove that this generalisation goes through here, but it should be possible to work it out from what we said in $\S \S 3-4$; recall that what is doing the work in those sections is the formal interaction between different parameters, not claims about what those parameters stand for or the content of the propositions in question. ${ }^{10}$

Secondly, and more speculatively, the argument in this paper may put some pressure on the distinction between assessment-sensitive and insensitive sentences generally. In $\S 3$ we saw that local relativism about knowledge attributions is straightforwardly incompatible with

\footnotetext{
${ }^{9} \mathrm{I}$ am very grateful to an anonymous referee for pressing me to spell out the wider implications of this paper, and especially for suggesting an argument along the lines of the second point below.

${ }^{10}$ I am grateful to a reviewer for reminding me that, if we follow Tim Williamson (2000: 34), then WOK can be understood as an instance of a more general schema, which we can call Ways of Factiving (WOF): for any subject $\mathrm{x}$ and any proposition $\mathrm{p}$, that $\mathrm{x} \varphi \mathrm{s}$ that $\mathrm{p}$ logically implies that $\mathrm{x}$ knows that $\mathrm{p}$, where $\varphi$ is any factive verb (e.g. 'realise', 'recognise' etc.). The reviewer suggests that if it is a consequence of my findings that RKA is incompatible not only with WOK, but also with WOF, then this immediately makes my findings less plausible. I disagree; if my findings generalise, as I suggest above they do, then we should expect consequences just like the incompatibility of WOF and RKA, and these consequences should be seen as an additional issue for RKA.
} 
WOK. In $\S 4$ we saw that adopting non-local relativism is a more promising (although I argued ultimately unsuccessful) route towards avoiding this result. If we assume that the reasoning in the previous paragraph is correct and the findings in this paper generalise, then we can say that whenever we have a proposition p expressed by a sentence $\Phi$ that we believe to be assessment-insensitive and a proposition q expressed by a sentence $\Psi$ that we believe to be assessment-sensitive, if we want to say that $\mathrm{p}$ logically implies $\mathrm{q}$, one way to secure this result is to revise our beliefs and claim that both $\Phi$ and $\Psi$ are assessment-sensitive. However, notice that every time we do this, we end up believing there are more assessment-sensitive sentences than we did previously. In other words, the price of attempting to preserve both WOK and RKA in this way, and by extension other views that rely on the relation of logical implication between propositions like $\mathrm{p}$ and $\mathrm{q}$, is a form of relativism that is, as it were, increasingly non-local. In light of this, we might wonder whether non-local relativism could spiral into what Crispin Wright (2008: 166-68) calls global relativism, i.e. relativism about all sentences. Our answer to this is likely to depend on our broader metaphysical and epistemological outlook. For example, a foundationalist might think that all propositions can be divided into sets $\mathrm{P}, \mathrm{Q}, \mathrm{R}$ and so on, where the members of $\mathrm{P}$ logically imply the members of Q, the members of Q logically imply the members of R and so on (see, e.g., Sosa 1980: 5). Given what we have said, a foundationalist might then be hard pressed to stop the move from non-local to global relativism. On the other hand, if one has a more sophisticated view in mind (e.g. Williams 1996), one will find it easier to say that only some propositions logically imply some others, and therefore there must be a limit to the number of sentences that might be 'converted' from assessment-insensitive to assessment-sensitive in this way. In any case, however these speculations turn out, again we find that semantic relativism invites more philosophical controversy than we currently see. I leave these more speculative elements for future work. ${ }^{11}$

\footnotetext{
${ }^{11}$ I wrote the first draft of this paper in 2014. Since then it has been through a long process of re-writes and refinements. I am grateful to everyone who has contributed to this process, especially several anonymous reviewers, Jessica Brown, David Liggins, Joel Smith, Graham Stevens and Thomas Uebel, and also to John MacFarlane and Crispin Wright for some early discussions that got me thinking about these issues. I am especially grateful to Rob Knowles, Thomas Uebel and Jack Winter for discussions (and sorry for pestering them for three years). Separate thanks to Rob, who also read a near-final draft and provided extensive feedback and encouragement.
} 


\section{Appendix I: Some simplifications and assumptions used throughout this paper}

The framework that we have worked with throughout this paper simplifies MacFarlane's view in several respects. In order to pre-empt any claims that we have worked with an inaccurate characterisation of relativism, below I explain which simplifications were made and why. I also note that the argument should generalise beyond MacFarlane's brand of relativism to other relativist frameworks.

Firstly, throughout this paper we have appealed to the notion of propositions. Relativists accept the standard assumptions about propositions. For example, John MacFarlane accepts that 'sentences are true or false because they express propositions that are true or false' and that '[propositions] are the contents of assertions and beliefs, and the things we call "true" or "false" in ordinary discourse' (MacFarlane 2014: 71). However, relativists are also careful to distance themselves from any commitments to the nature of propositions that are not essential to their theory (see e.g. MacFarlane 2014: 72). We can follow suit; although we have worked with propositions, nothing we say should be taken to commit us to claims about their nature.

Secondly, I sketched the relativist framework in $\$ 2$ on the basis of David Kaplan's (1989) well-known semantic framework for the analysis of indexical expressions and MacFarlane's (2014: Ch. 4) modifications to this framework. Kaplan's framework makes use of four key notions: (i) contexts, understood to represent possible occasions of utterance of a sentence and defined as sequences of at least individuals, times, locations and possible worlds (Kaplan 1989: 543); (ii) characters, defined as functions from contexts to propositions (Kaplan 1989: 505-506); (iii) propositions (contents in Kaplan's terminology), defined as functions from circumstances of evaluation to truth-values (Kaplan 1989: 500-501); and (iv) circumstances of evaluation, defined as sequences of the worlds and times parameters of the contexts and a parameter for assignments of values to variables, where each parameter can vary independently of another (Kaplan 1989: 502-504).

We need to note three things in relation to this framework. Firstly, MacFarlane (2014: Ch. 3) defines several key notions - most relevantly for our purposes, the notion of logical implication - using David Lewis' (1980) rather than Kaplan's framework. Lewis' framework works only with sentences and open formulas, not propositions. However, we have already seen that MacFarlane accepts that sentences have truth-values because they express propositions and propositions have truth-values. Moreover, his discussion of propositions indicates that what he has to say about sentences extends mutatis mutandis to 
propositions (see MacFarlane 2014: Ch. 4, especially 90-92). In light of this, in §3 I extended the relevant claims MacFarlane makes about sentences - in particular, his definition of logical implication - to propositions. Secondly, MacFarlane (2014: 58-60) distinguishes semantics proper from what he calls post-semantics. Roughly put, semantics proper deals with the compositional aspect of semantics, i.e. the contribution the meanings of sentential constituents make to the meanings of the sentences in which they occur. Post-semantics deals with the aspect of semantics which, as MacFarlane (2014: 57) puts it, has 'direct pragmatic relevance', i.e. the aspect which tells us whether a sentence is true or false as used on a particular occasion. The distinction affects the way one should define truth for sentences and propositions (see e.g. MacFarlane 2014: 67, 91), but it is not important for our purposes. In light of this, I sketched the relativist framework in $\S 2$ in a way that is consistent with the distinction, but that ignores it. Thirdly, we have already noted that Kaplan treats contexts as sequences of at least individuals, times, locations and possible worlds, and circumstances of evaluation as sequences of the worlds and times parameters of the contexts and a parameter for assignments of values to variables. We did not need such a rich picture of contexts and circumstances for our purposes; in particular, I did not countenance individuals, times, locations or assignments in my sketch of the relativist framework in $\S 2$.

Next, note that there is a distinction between what Max Kölbel (2008: 18-20) calls moderate and radical relativism, or what MacFarlane (2014: 88) calls non-indexical contextualism and relativism. The difference is roughly this: whereas moderate relativists add unorthodox parameters (i.e. parameters besides possible worlds and times) to circumstances of evaluation and say that the values of these parameters are determined by contexts of utterance, radical relativists say that the values of both orthodox and unorthodox parameters of circumstances of evaluation may be determined by so-called contexts of assessment. Radical relativism is the most popular relativist account of the semantics of knowledge attributions (MacFarlane 2005; MacFarlane 2014: Ch. 8), so it is the view I have worked with throughout this paper. However, as we saw in $\S 3$ and $\S 4$, my claim that WOK an RKA are incompatible depends mainly on the idea that the proposition expressed by a knowledge attribution and the propositions expressed by some other sentences change truthvalues with respect to different parameters of the circumstances of evaluation, where the values of these parameters vary independently of each other. This idea is common to both moderate and radical relativists, so what I had to say about radical relativism about knowledge attributions should also apply to moderate relativism about knowledge attributions. 
Finally, MacFarlane (e.g. 2014: 49-52) points out that the key aspect of radical relativism is not the addition of unorthodox parameters to circumstances of evaluation, but the idea that the values of the parameters of circumstances of evaluation may be determined by contexts of assessment. However, we just noted that radical relativists think that the proposition expressed by a knowledge attribution changes truth-values with respect to an unorthodox parameter of circumstances of evaluation the value of which is determined by contexts of assessment, so, given that we are focusing on knowledge attributions here, it does not matter if we ignore the thought that the values of orthodox parameters of circumstances of evaluation may be determined by contexts of assessment.

\section{Appendix II: Demonstration of a point in $§ 4$}

In $\S 4$ we argued that there are good reasons to expect the truth-values of the propositions expressed by a knowledge attribution and a perception sentence to vary with respect to different parameters determined by contexts of assessment, where the values of these parameters vary independently of each other. On this basis, we inferred that we should expect the truth-values of these propositions not to co-vary in the right way, and therefore for non-local relativism to be incompatible with WOK. Here I demonstrate that this inference is sound.

Make all the assumptions we made in $\S 3$, except now suppose that both sentences (1) and (2) are assessment-sensitive, the truth value of $\mathrm{ALICE}_{1}$ varies with what we will call visual standards $\mathrm{v}_{\mathrm{a}}$ determined by contexts of assessment, but not epistemic standards, and the truth-value of $\mathrm{ALICE}_{2}$ varies with epistemic standards $e_{a}$ determined by contexts of assessment, but not visual standards. (This is consistent with the argument in $\$ 4$ that the truth-values of $\mathrm{ALICE}_{1}$ and $\mathrm{ALICE}_{2}$ vary with respect to different parameters of circumstances of evaluation; there we also briefly touched on the notion of visual standards). Our semantic framework in $\$ 2$ can be straightforwardly extended to accommodate this: circumstances of evaluation can be understood as triples of worlds of the context of utterance and visual and epistemic standards determined by contexts of assessment: $\left\langle\mathrm{w}_{\mathrm{c}}, \mathrm{v}_{\mathrm{a}}, \mathrm{e}_{\mathrm{a}},\right\rangle$. However, for the sake of the argument, let us assume that if $\mathrm{ALICE}_{1}$ and $\mathrm{ALICE}_{2}$ are true, they are true at all possible worlds, and therefore drop worlds from our model. We know that it is the interaction between the values of $v_{a}$ and $e_{a}$ that needs to do the work in order for the argument in $\S 4$ to go through, and dropping worlds from our model will help to keep things as simple as possible. 
Now imagine we are working in a toy semantic model that has four, and only four, circumstances of evaluation: $\left\langle\mathrm{v}_{\mathrm{a} 1}, \mathrm{e}_{\mathrm{a} 1}\right\rangle,\left\langle\mathrm{v}_{\mathrm{a} 1}, \mathrm{e}_{\mathrm{a} 2}\right\rangle,\left\langle\mathrm{v}_{\mathrm{a} 2}, \mathrm{e}_{\mathrm{a} 1}\right\rangle,\left\langle\mathrm{v}_{\mathrm{a} 2}, \mathrm{e}_{\mathrm{a} 2}\right\rangle$. Assume ALICE $_{1}$ is true at $\left\langle v_{\mathrm{a} 1}, \mathrm{e}_{\mathrm{a} 1}\right\rangle$. Then ALICE $_{1}$ is also true at $\left\langle\mathrm{v}_{\mathrm{a} 1}, \mathrm{e}_{\mathrm{a} 2}\right\rangle$ and false at $\left\langle\mathrm{v}_{\mathrm{a} 2}, \mathrm{e}_{\mathrm{a} 1}\right\rangle$ and $\left\langle\mathrm{v}_{\mathrm{a} 2}, \mathrm{e}_{\mathrm{a} 2}\right\rangle$. Assume that $\mathrm{ALICE}_{2}$ is likewise true at $\left\langle\mathrm{v}_{\mathrm{a} 1}, \mathrm{e}_{\mathrm{a} 1}\right\rangle$. Then $\mathrm{ALICE}_{2}$ is also true at $\left\langle\mathrm{v}_{\mathrm{a} 2}, \mathrm{e}_{\mathrm{a} 1}\right\rangle$ and false at $\left\langle\mathrm{v}_{\mathrm{a} 1}, \mathrm{e}_{\mathrm{a} 2}\right\rangle$ and $\left\langle\mathrm{v}_{\mathrm{a} 2}, \mathrm{e}_{\mathrm{a} 2}\right\rangle$. This is represented in Table 2:

Table 2: Circumstances of evaluation at which ALICE 1 and ALICE 2 are true and circumstances at which they are false in the non-local relativism example

\begin{tabular}{|c|c|c|}
\cline { 2 - 3 } \multicolumn{1}{c|}{} & $\mathbf{v a 1}$ & $\mathbf{v a}^{1}$ \\
\hline $\mathbf{e}_{\mathbf{a} 1}$ & $\mathrm{ALICE}_{1}$ & ALICE $_{2}$ \\
& ALICE $_{2}$ & \\
\hline $\mathbf{e}_{\mathbf{a} 2}$ & ALICE $_{1}$ & \\
\hline
\end{tabular}

There is a circumstance of evaluation, viz. $\left\langle\mathrm{v}_{\mathrm{a} 1}, \mathrm{e}_{\mathrm{a} 2}\right\rangle$, at which $\mathrm{ALICE}_{1}$ is true but $\mathrm{ALICE}_{2}$ is false, so ALICE 1 does not logically imply ALICE 2 .

\section{References}

Brown, J. (2006). Contextualism and Warranted Assertibility Manoeuvres. Philosophical Studies, 130/3: 407-35.

Chisholm, R. (1989). Theory of Knowledge. Foundations of Philosophy, 3rd ed. PrenticeHall, Inc.

Cohen, S. (1999). Contextualism, Skepticism, and the Structure of Reasons. Nô̂s, 33/13: 5789.

Daly, C., \& Liggins, D. (2011). Deferentialism. Philosophical Studies, 156/3: 321-37.

DeRose, K. (2009). The Case for Contextualism: Knowledge, Skepticism, and Context, Vol. 1. Oxford University Press: Oxford.

Dretske, F. (1970). Epistemic Operators. The Journal of Philosophy, 67/24: 1007-23.

Edgington, D. (1995). On Conditionals. Mind, 104/414: 235-329. 
Goldman, A. (1976). Discrimination and Perceptual Knowledge. The Journal of Philosophy, 73/20: 771-91.

Hawthorne, J. (2004). Knowledge and Lotteries. Oxford University Press: Oxford.

Kaplan, D. (1989). Demonstratives: An Essay on the Semantics, Logic, Metaphysics, and Epistemology of Demonstratives and Other Indexicals. Almog J., Perry J., \& Wettstein H. (eds) Themes from Kaplan, pp. 481-563. Oxford University Press: Oxford.

Kölbel, M. (2008). Motivations for Relativism. García-Carpintero M. \& Kölbel M. (eds) Relative Truth, pp. 1-38. Oxford University Press: Oxford.

Kolodny, N. \& MacFarlane, J. (2010). Ifs and Oughts. The Journal of Philosophy, 107/3: $115-43$.

Lewis, D. (1980). Index, Context, and Content. Kanger S. \& Öhman S. (eds) Philosophy and Grammar, pp. 79-100. Springer: Dordrecht.

MacFarlane, J. (2005). The Assessment Sensitivity of Knowledge Attributions. Gendler T. \& Hawthorne J. (eds) Oxford Studies in Epistemology: Volume 1, pp. 197-233. Oxford University Press: Oxford.

—. (2014). Assessment Sensitivity: Relative Truth and Its Applications. Oxford University Press: Oxford.

Moore, G. E. (1953). Some Main Problems of Philosophy. London: George Allen \& Unwin Ltd.

Russell, B. (1948). Human Knowledge: Its Scope and Limits. Simon and Schuster: New York.

Sosa, E. (1980). The Raft and the Pyramid: Coherence versus Foundations in the Theory of Knowledge. Midwest Studies In Philosophy, 5/1: 3-26.

Stephenson, T. (2007). Indicative Conditionals Have Relative Truth Conditions. Proceedings from the Annual Meeting of the Chicago Linguistic Society, 43/1: 231-42.

Tarasov, L. (2013). Contextualism and Weird Knowledge. The Philosophical Quarterly, 63: 252: 565-575.

—. (2014). The Semantics of Knowledge Attributions: A Defence of Moderate Invariantism. The University of Manchester: Manchester ( $\mathrm{PhD}$ thesis).

Turri, J. (2010). Does Perceiving Entail Knowing? Theoria, 76/3: 197-206.

Williams, M. (1996). Unnatural Doubts: Epistemological Realism and the Basis of Scepticism. Princeton University Press: Princeton.

Williamson, T. (2000). Knowledge and its Limits. Oxford University Press: Oxford.. 
Wright, C. (2008). Relativism about Truth Itself: Haphazard Thoughts about the Very Idea. García-Carpintero, M. \& Kölbel, M. (eds) Relative Truth, pp. 157-85. Oxford University Press: Oxford. 